\title{
“Características epidemiológicas y clínicas de las lesiones escamosas intraepiteliales cervicales (LEIC) en el Hospital Departamental de Sabanalarga"
}

\author{
Raquel Fernández Amador*; Robinson Fernández Mercado**; Guillermo Acosta Osio***; Alfredo Barraza Támara****
}

\section{RESUMEN}

Las lesiones escamosas intraepiteliales (LEIC) siguen siendo un problema de salud importante para las mujeres de todo el mundo, por lo que se realizó un estudio para determinar las características epidemiológicas y clínicas más frecuentes de esta patología.

Se analizaron retrospectivamente las historias clínicas de 88 pacientes con diagnóstico de LEIC del Hospital Departamental de Sabanalarga (Atlántico) entre Septiembre de 1994 a Septiembre de 1997 . EI $40 \%$ era procedente de Sabanalarga, mientras el $60 \%$ proce día de diversos municipios del Atlántico, Córdoba y Bolívar, siendo la prevalencia de $1.72 \%$.

Se encontró que el $67 \%$ de las pacientes con LEIC correspondía a mujeres entre los 30-50 años, sólo el $1 \%$ era menor de 16 años. El $61 \%$ de las pacientes iniciaron relaciones sexuales antes de los 20 años, sin embargo como no hubo grupo control en este estudio, estos datos no son $100 \%$ confiables. El $64 \%$ declararon ser casadas, $19 \%$ solteras y $17 \%$ unión libre. Llama la atención que el $72 \%$ eran monógamas. El diagnóstico citológico-colposcópico más frecuente fue LEIC alto grado (NIC II-III) (65\%), datos corroborados por biopsia.

El tratamiento más empleado fue la histerectomía (74\%), seguido por la conización (13\%). El $55 \%$ de las pacientes no tuvo control citológico post-tratamiento.

Palabras claves: Lesiones escamosas intraepiteliales cervicales - colposcopia en L.E.I.C. - colposcopia cáncer de cervix.

\section{SUMMARY}

The cervical intraepithelial squamous lesions (CIL) are still a health problem to women around the world therefore an investigation was made to determine the epidemiological features most frequent of this pathology.

88 patients were analyzed retrospectively with diagnosis of cervical intraepithelial squamous lesions of the Hospital departamental de Sabanalarga (Atlántico), between September 1994 to September 1997.40\% come from Sabanalarga, unhide $60 \%$ preceded for several towns from Atlántico, Córdoba and Bolívar, being the prevalence $1.72 \%$.

The results founded $67 \%$ of the patients with cervical intraepithelial squamous lesion correspond to women between $30-50$ years old, only $1 \%$ of the were younger than 16 years old $61 \%$ of the patients began sexual activities before 20 years old nevertheless this data is not to be trusted because there was no group control on this study. $64 \%$ declared themselves to be married, $19 \%$ singles and $7 \%$ free union. $72 \%$ were monogamies. The colposcopic ant citologic diagnosis most frequent was cervical intraepithelial squamous lesions high grade (NIC IIIII) $65 \%$ this information was confirmed by biopsy.

The most need treatment was hysterectomy (74\%) followed by conization (13\%). 555 of the patients did not have a post cytologic treatment control.

Key words: Cervical intraepithelial squamous lesions, cervical cancer, colposcopy, colposcopy in cervical intraepithelial squamous.

\section{Introducción}

Las lesiones escamosas intraepiteliales cervicales (LEIC) constituyen un problema de salud, para las mujeres de todo

\footnotetext{
* Ginecóloga-Obstetra- Universidad Metropolitana.

** Ginecólogo-Oncólogo. Hospital de Sabanalarga.

*** Director Dpto. Ginecología y Obstetricia. Universidad Metropolitana.

**** Coordinador Postgrado Ginecología-Obstetricia. Universidad Metropolitana.
}

el mundo, por eso hoy día se cuenta con programas de detección precoz para el cáncer cervical como lo es la citología cervico-vaginal (1). Sin embargo en los países subdesarrollados como no existen estos programas bien establecidos, un alto porcentaje de mujeres dejan de ser estudiadas, por ello con este trabajo se pretende identificar las características epidemiológicas y clínicas de las lesiones escamosas intraepiteliales cervicales (LEIC) en el Hospital Departamental de Sabanalarga, lo cual incluye determinar la prevalencia de estas lesiones premalignas del cérvix, establecer la correlación que existe entre citología-colposcopia y 
biopsia y que métodos terapéuticos se utilizaron para su manejo y posterior seguimiento. Es importante concientizar a toda la comunidad en general y al personal que labora en salud sobre los factores de riesgo de esta patología, formas de diagnóstico, tratamiento y posterior seguimiento.

\section{Materiales y métodos}

Se realizó un estudio retrospectivo en donde se revisaron las historias clínicas de las pacientes con diagnóstico de LEIC que acudieron a la consulta de ginecología-oncológica del Hospital Departamental de Sabanalarga (Atlántico) entre Septiembre de 1994 y Septiembre de 1997.

Los datos obtenidos de las historias clínicas fueron ordenados y tabulados, determinando la frecuencia de cada uno de los factores de riesgo relacionados con LEIC. Así mismo se tuvo en cuenta el diagnóstico de LEIC más frecuente en el reporte citológico-colposcópico y anatomopatológico definitivo y el tipo de tratamiento más empleado.

\section{Resultados y discusión}

Se revisaron 5130 historias clínicas, de las cuales se estudiaron 88 pacientes con diagnóstico de LEIC, siendo la prevalencia de $1.72 \%$.

El $40 \%$ de las pacientes procedía de Sabana larga, mientras que el resto procedía de diversos municipios del Atlántico e incluso de Córdoba, Bolívar y Magdalena (tabla 1). En este estudio el grupo etáreo de mayor frecuencia de LEIC comprende pacientes entre los 30 y 50 años lo que ratifica que el cáncer cervical en la tercera y cuarta década de la vida es más frecuente en este grupo de edad (1). Sólo 8 pacientes de las 88 que se estudiaron con edad comprendida entre los 21 y 29 años presentaban LEIC (gráfica 1).

Cabe anotar que 56 pacientes $(64 \%)$ declararon ser casadas, mientras que 17 pacientes $(19 \%)$ eran solteras (gráfica 2). Sin embargo el estado civil de las mujeres no constituye un factor de riesgo como tal, ya que para desarrollar LEIC deben existir otros factores asociados como: edad del primer coito, edad del primer embarazo y número de compañeros sexuales (1).

En cuanto a la paridad, se observa que más de la mitad de las pacientes (55\%) tuvieron entre 2 a 3 gestaciones, sin embargo como este estudio se realizó con un pequeño número de pacientes no se puede demostrar lo escrito en la literatura "entre mayor es la multiparidad, mas riesgo tiene la paciente de desarrollar LEIC" (4), ya que en este estudio sólo el $16 \%$ de las pacientes tenían entre 4 y 5 gestaciones y sólo el 27\% tenían más de 5 hijos (tabla 2).

Hoy en día se habla mucho de la actividad sexual de la mujer y su relación con el riesgo de desarrollar LEIC, en este estudio solamente el $27 \%$ de las pacientes manifestaron tener 2 o más compañeros sexuales, mientras que la mayoría de las pacientes con LEIC (72\%) declararon tener un solo compañero sexual (tabla 3 ) no existiendo correlación con la literatura. Sin embargo, cabe anotar que el dato del número de compañeros sexuales no es confiable en este estudio, seguramente por el impacto psicológico que este tipo de preguntas produce en la comunidad femenina sobre todo las que pertenecen a un estatus sociocultural bajo.

Como se observa en la gráfica 3 , el $61 \%$ de las pacientes con LEIC iniciaron sus relaciones sexuales antes de los 20 años, mientras que sólo el $26 \%$ las iniciaron después de los 20 años, sin embargo como no hubo grupo control en este estudio no se puede ratificar lo escrito en la literatura acerca del inicio de las relaciones sexuales antes de los 20 años y el riesgo elevado de estas pacientes para desarrollar LEIC (2).

El sistema empleado para la detección de LEIC en el HDS fue el de Papanicolau (tabla 4). Cabe anotar que el

\section{Gráfica 1}

FRECUENCIA DE PRESENTACION DE LEIC SEGUN EDAD

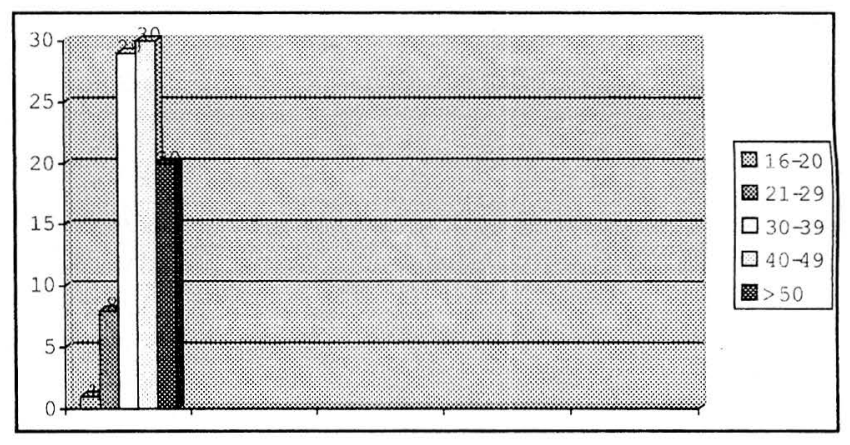

Tabla 1

PROCEDENCIA DE PACIENTES CON LEIC EN EL H.D.S.

\begin{tabular}{|lcc|}
\hline Procedencia & $\begin{array}{c}\text { No. De } \\
\text { Pacientes }\end{array}$ & $\begin{array}{c}\text { Porcentaje } \\
(\boldsymbol{\%})\end{array}$ \\
Sabanalarga & 36 & 40 \\
Barranquilla (H.U.M.) & 14 & 16 \\
Baranoa & 1 & 1.2 \\
Campo de la Cruz & 1 & 1.2 \\
Calamar & 3 & 3.4 \\
Candelaria & 2 & 2.3 \\
Ciénaga & 2 & 2.3 \\
Magangué & 3 & 3.4 \\
Montería & 1 & 1.2 \\
Malambo & 2 & 2.3 \\
Tubará & 1 & 1.2 \\
Luruaco & 7 & 8 \\
Arenal & 1 & 1.2 \\
Manatí & 2 & 2.3 \\
Piojó & 1 & 1.2 \\
Polonuevo & 1 & 1.2 \\
Ponedera & 2 & 2.3 \\
Puerto Colombia & 2 & 2.3 \\
Repelón & 4 & 5 \\
Suán & 2 & 2.3 \\
TOTAL & 88 & 100 \\
\hline
\end{tabular}


Gráfica 2

ESTADO CIVIL VS. LEIC

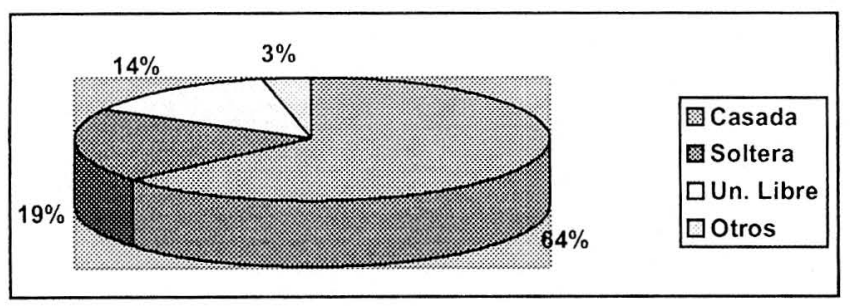

Tabla 2

No. PARIDAD VS. LEIC EN EL HDS

\begin{tabular}{|lcc|}
\hline Paridad & No. Pacientes & $\%$ \\
1 & 2 & 2 \\
$2-3$ & 48 & 55 \\
$4-5$ & 14 & 16 \\
$>5$ & 24 & 27 \\
TOTAL & 88 & 100 \\
\hline
\end{tabular}

Gráfica 3

INICIO DE RELACIONES SEXUALES VS. LEIC

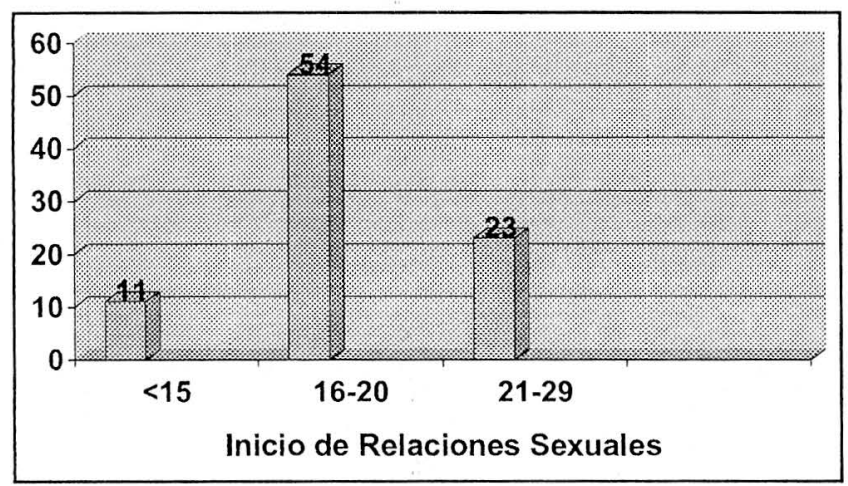

Tabla 3

No. DE COMPAÑEROS SEXUALES Y SU RELACION CON LEIC

\begin{tabular}{|lcc|}
\hline Compañeros & $\begin{array}{c}\text { No. De } \\
\text { pacientes }\end{array}$ & Porcentaje $(\%)$ \\
sexuales & 63 & 72 \\
1 & 24 & 27 \\
$2-3$ & 1 & 1 \\
$4-5$ & 88 & 100 \\
TOTAL & & \\
\hline
\end{tabular}

Gráfica 4

DIAGNOSTICO COLPOSCÓPICO Y LEIC / HDS

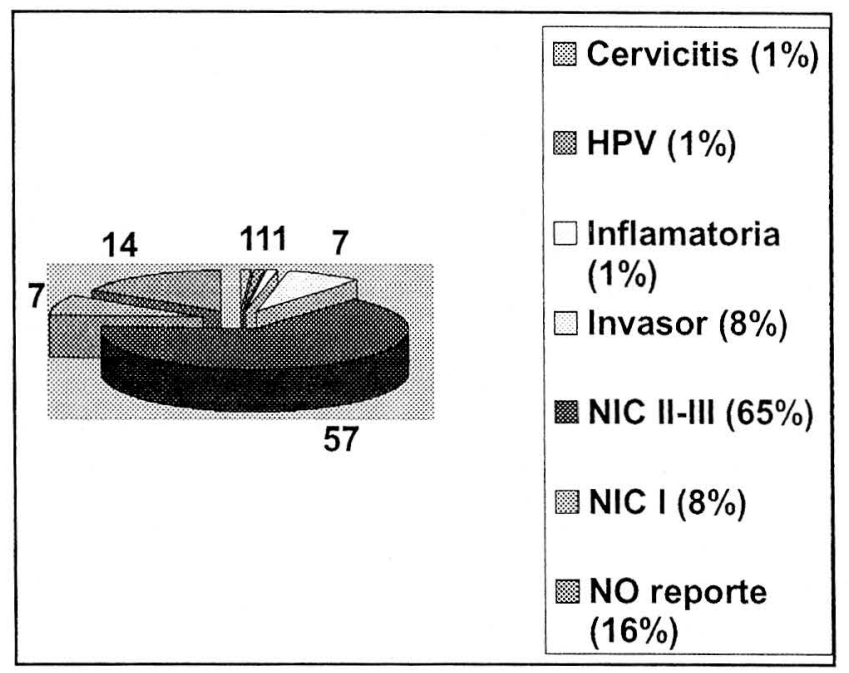

Gráfica 5

TRATAMIENTO DE LEIC EN EL HDS

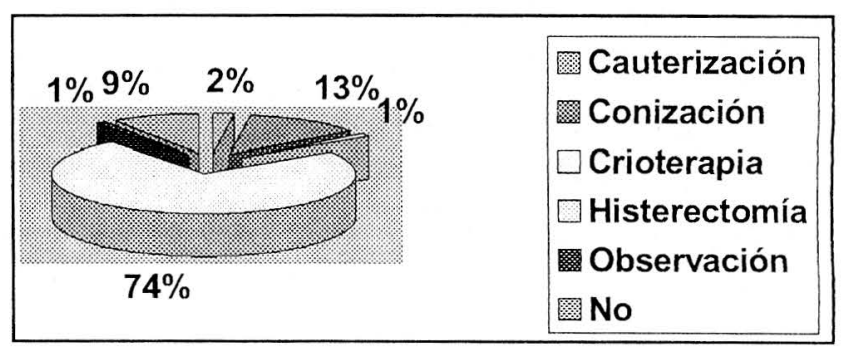

Gráfica 6

SEGUIMIENTO DE LEIC EN EL HDS

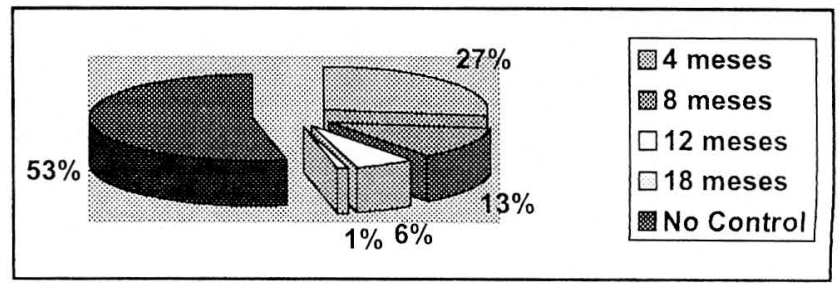

Tabla 4

No. DE CITOLOGIAS POSITIVAS EN EL HDS

\begin{tabular}{|lcc|}
\hline Citologías & No. Pacientes & Porcentaje (\%) \\
PAP 1 & 1 & 1 \\
PAP II & 16 & 18 \\
PAP III & 53 & 61 \\
PAP IV & 9 & 10 \\
PAP V & 9 & 10 \\
TOTAL & 88 & 100 \\
\hline
\end{tabular}


$80 \%$ de las pacientes tenían reporte citológico anormal, mientras que 17 (20\%) presentaban citologías normales (PAP I -PAP II), sin embargo es importante recordar que la citología presenta un $50 \%$ de falsos negativos en su interpretación, cifra que puede reducirse a un 5\% al utilizar técnicas adecuadas para la toma de muestra (5).

El diagnóstico colposcópico cada día cobra más importancia, si se tiene en cuenta que la colposcopia nos ayuda a tomar la biopsia del sitio más sospechoso cuando no existe una lesión cervical aparente (3). En este estudio vale la pena mencionar el alto porcentaje de LEIC de alto grado (NIC II-NIC III), (65\%), diagnosticado por colposcopia (gráfica 4).

El tratamiento para las lesiones escamosas intraepiteliales cervicales (LEIC), se realiza de acuerdo a las condiciones y deseos de la paciente, así como la experiencia del médico (1). En este estudio el tratamiento más empleado fue la histerectomía abdominal ampliada clase 1 (HAA), teniendo en cuenta que la mayoría de las pacientes son mayores de edad y con paridad definida (gráfica 5).

Es llamativo que más de la mitad de las pacientes que se realizan la citología no lo hacen con los criterios de seguimiento establecidos (gráfica 6). De las 41 pacientes que acudieron a control citológico sólo 24 lo hicieron a los 4 meses y el resto en los meses siguientes. Esto significa que la mayoría de las pacientes desconocen el potencial de malignidad de las lesiones precursoras de cáncer cervical, así mismo se necesita del apoyo Psicológico y de la colaboración de las trabajadoras sociales para rescatar las pacientes que se pierden de la institución y llevar así un seguimiento post-tratamiento adecuado.

\section{Conclusiones}

- Es el primer estudio para esta patología realizado fuera de la capital del departamento del Atlántico.

- Esta entidad se sigue presentando especialmente durante la edad reproductiva de la mujer con paridad entre 2 y 3 en su mayoría.

- Más de la mitad iniciaron relaciones sexuales antes de los 20 años de edad, sin embargo el número de compañeros sexuales no es confiable en muchas ocasiones, tal vez por el impacto psicológico que este tipo de preguntas produce en la población femenina de estrato sociocultural bajo.

- El antecedente de tabaquismo no fue importante en este estudio como factor de riesgo para LEIC.
MANEJO DE LA CITOLOGIA VAGINAL ANORMAL* SIN LESION CLINICA APARENTE

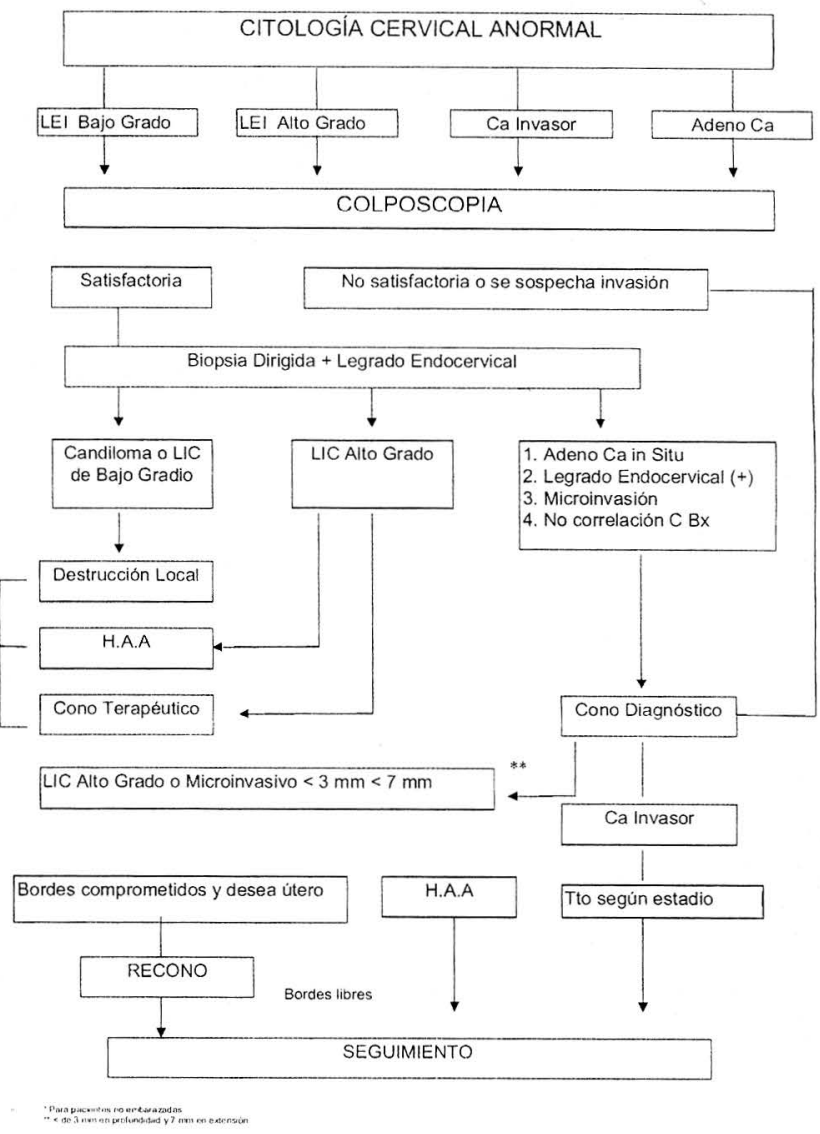

- El diagnóstico citológico y colposcópico más frecuente fue LEIC de alto grado (NIC II - NIC III) (65\%), dato corroborado posteriormente por histopatología.

- El tratamiento más usado fue la histerectomía, seguida de conización en 11 pacientes de las cuales en 7 casos fue diagnóstica, en 2 casos diagnóstica y terapéutica y en 2 terapéuticas.

- Actualmente siguen existiendo pacientes a quienes no se le realizan biopsias posterior a histerectomía o conización (26\%). Probablemente por escasez de recursos económicos.

- Llama la atención en este estudio que sólo $41 \%$ tuvieron control citológico post-tratamiento en forma irregular.

\section{BIBLIOGRAFIA}

1. Disaia Creasman. Oncología Ginecología Clínica. 5a edición. 1997

2. W. Jones/ G.S Jones. Tratado de Ginecología de Novak. 11a edición. 1996.

3. Manual de normas de Ginecología y Obstetricia. 2a edición 1991. Guillermo Acosta O.

4. González M. Oncología Ginecología. Edición 1994.

5. Manual de normas del manejo del cáncer genital femenino. Gilberto Martínez M. Edición 1992.

6. Revista Colombiana de Obstetricia y Ginecología. Vol 45. $\mathrm{N}^{\circ} 1$. Enero 1994
7. Clínicas de Norteamérica. Dx y Tto de la Neoplasia Intraepitelial Cervical. Vol 3. 1995.

8. Manual de colposcopia para ginecólogos. Edición 1995.

9. De Palo, Giuseppe MD. Colposcopia y Patología del Tracto Genital Inferior. Edición 1996.

10. Torres S. Protocolo de manejo en patología Gineco-oncológica del Hospital Universitario del Valle. Edición 1995. 